\title{
Preface for Feature Topic on Intelligent Safety for CAVs
}

\author{
Jun $\mathrm{Li}^{1} \cdot$ Hong Wang ${ }^{1}$
}

Accepted: 25 June 2021 / Published online: 2 August 2021

(c) China Society of Automotive Engineers (China SAE) 2021

Connected and automated driving confronts critical complex traffic scenarios and safety, which attracts increasing attention from academia and industry. To enhance the intelligent safety for connected and automated vehicles (CAVs), including functional safety, safety of the intended functionality (SOTIF), and cybersecurity, efforts are required to seek solutions from fundamental theory, guarantee framework and general/systematic point of view. This feature topic aims to provide a platform for academia, industry, and policymakers, on which they could present the latest research and engineering experience in developing and applying novel technologies. The topics cover SOTIF, cybersecurity, artificial intelligence for CAV safety, risk assessment-based vehicle path planning and decision-making under uncertain environments.

\section{Paper Highlight}

1. Most of the SOTIF issues happen in the perception layer, where object detection algorithm plays a key role. The uncertainty and randomness of the object detection algorithm might cause fatal accidents in autonomous driving. Capturing uncertainty in object detection is indispensable for safe autonomous driving. "Uncertainty Evaluation of Object Detection Algorithms for Autonomous Vehicles" by Jun Li et al. from Tsinghua University presents a quantitative evaluation method for the uncertainty of the object detection algorithms which are verified by the Chinese SOTIF scenario dataset, and it demonstrates the potential to monitor object detection algorithm online and provides a valuable reference for readers who are interested in the uncertainty of artificial intelligence for vehicles.

2. The increasing amount of CAVs has brought many unprecedented automotive cybersecurity threats, which may cause

Hong Wang

hong_wang@tsinghua.edu.cn

1 School of Vehicle and Mobility, Tsinghua University, Beijing, China privacy breaches, personal injuries and even national security issues. "A Systematic Risk Assessment Framework of Automotive Cybersecurity" by Yunpeng Wang et al. from Beihang University proposes a systematic risk assessment framework of automotive cybersecurity that comprises a specific risk assessment process and systematic risk assessment methods, which applies to all phases of the vehicle lifecycle. The proposed assessment framework helps to meet automotive cybersecurity requirements.

3. "Cyber-Attack Detection for Autonomous Driving Using Vehicle Dynamic State Estimation" by Chen Lv et al. from Nanyang Technology University proposes a cyberattack detection approach for autonomous vehicles based on secure estimation of vehicle states. This paper presents a case study of a vehicle localization system under attack, which displays great application potential.

4. "Human Performance in Critical Scenarios as a Benchmark for Highly Automated Vehicles" by Laura Quante et al. from the German Aerospace Center uses human performance as a benchmark to assess the safety performance of highly automated vehicles, which narrows the gap of the recording and description of human performance between uncritical and critical scenarios. This paper contributes to the ongoing development of testing and verification methods for highly automated vehicles.

5. Autonomous driving is a challenging task in complex traffic scenarios, as uncertainties in dynamic and physical parameters would cause safety issues. "Path-Following Control of Autonomous Vehicles Considering Coupling Effects and Multi-source System Uncertainties" by Yinong $\mathrm{Li}$ et al. from Chongqing University proposes methods to solve different types of coupling in vehicle dynamics, and robust control methods to deal with the uncertainties in dynamic and physical parameters. This paper provides effective solutions to tackle coupling effects and system uncertainties of vehicles in path-tracking control.

The publication of this feature topic would not be achieved without the meticulous work and joint efforts of the authors, reviewers, editors and the editorial office of Automotive 
Innovation, and we would like to express gratitude to all of them for their hard work and support. We are also indebted to the support from Tsinghua University, University of Electronic Science and Technology of China, Nanyang Technology University, Carnegie Mellon University and University of Waterloo.

Heartily hope this feature topic can inspire scientific ideas and promote research in the field of intelligent safety.

\section{Guest Editors}

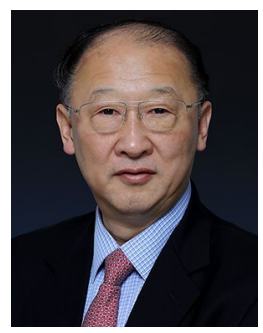

Jun Li received his $\mathrm{PhD}$ degree in vehicle engineering from Jilin University. He is currently an academician of the Chinese Academy of Engineering, a professor at School of Vehicle and Mobility with Tsinghua University, president of the China SAE, director of the expert committee of China Industry Innovation Alliance for the Intelligent and Connected Vehicles. His research interests include internal combustion engine, electric drive systems, electric vehicles, intelligent and connected vehicles.

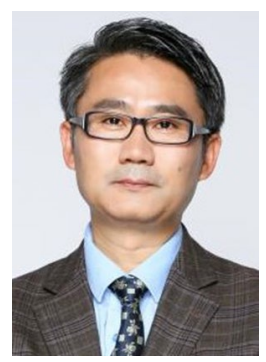

Hong Cheng is currently a full professor at University of Electronic Science and Technology of China. He is a Leading Talent in Science and Technology Innovation of National Ten Thousand Plan. He received his PhD degree from Xi' an Jiaotong University in 2003 and held a postdoctoral position at Carnegie Mellon University in the USA from 2006 to 2009. His research fields include computer vision, machine learning, robotics and intelligent vehicles. He has published 120 papers in top international conferences and journals.

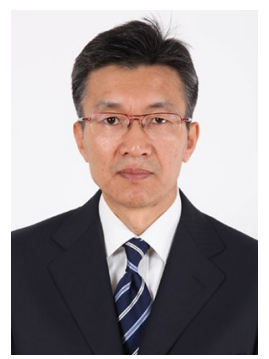

Shaobo Qiu is currently a research professor at Machine Intelligence Institute, University of Electronic Science and Technology of China. He was the director of Intelligent Driving and Passive Safety Technology of FAW R\&D Center, as well as the chief technology representative in Germany (1994) in FAW. He also used to be the representative of FAW R\&D Center and Qiming Information Technology Company in Silicon Valley, USA (2016). He has been the deputy chairman of the Safety Technology Committee of China SAE, the chairman of the China Expert Group of the WP-29/GRSP in United Nations ECE, the member of the GESP expert group in the OICA and member of AQSIQ Product Defect Identification Expert Group. He serves as the editorial board member for the Journal of Automotive Safety and Energy. His research focuses on automobile body test, CAE analysis, passive safety design and intelligent driving system design. He published one book, titled Automobile Crash Safety Engineering (2016).

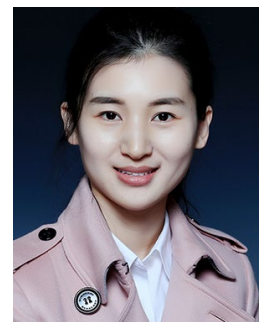

Hong Wang is currently a research associate professor at Tsinghua University. From the year 2015 to 2019 , she was working as a research associate of Mechanical and Mechatronics Engineering with the University of Waterloo. She received her PhD degree in Beijing Institute of Technology in China in the year 2015. Her research focuses on the risk assessment and crash mitigation-based decision making during critical driving scenarios, ethical decision making for autonomous vehicles, safety of the intended functionality (SOTIF). She has published over 50 papers on top international journals.

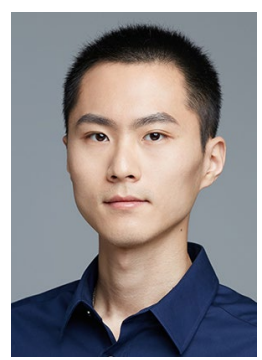

Chen Lv is an assistant professor at the School of Mechanical and Aerospace Engineering and Cluster Director in Future Mobility Solutions, Nanyang Technological University (NTU), Singapore. He received his $\mathrm{PhD}$ degree at Tsinghua University, in 2016. He was a joint $\mathrm{PhD}$ at EECS Dept., University of California, Berkeley, during 2014-2015, and worked as a research fellow at Cranfield University, UK, during 2016-2018. He joined NTU and founded the AutoMan Research Group in June 2018. His research focuses on automated driving and human-machine systems, where he has contributed 2 books, over 100 papers and obtained 12 granted patents.

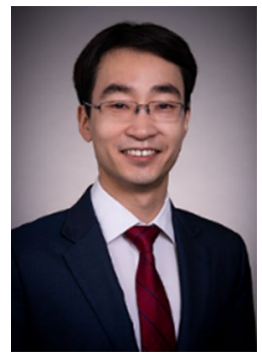

Ding Zhao received his PhD degree from the University of Michigan, Ann Arbor, MI, USA, in 2016. He is currently an assistant professor with the Department of Mechanical Engineering, Carnegie Mellon University. His research focuses on the intersection of statistical machine learning, robotics and optimal design, with applications on autonomous vehicles and connected/smart cities, spanning many aspects of these intelligent physical systems, such as safety, energy efficiency, human-machine interaction and cybersecurity.

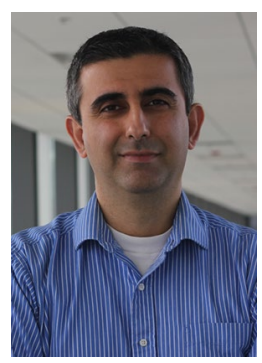

Ehsan Hashemi received his $\mathrm{PhD}$ in Mechanical and Mechatronics Engineering in 2017 from University of Waterloo. He is currently an assistant professor at the Department of Mechanical Engineering, University of Alberta, and principal investigator of the Networked Optimization, Diagnosis, and Estimation (NODE) lab. Before joining University of Alberta, he was a visiting professor at KTH Royal Institute of Technology and a research assistant professor at the University of Waterloo. His research interests are robotics, control theory, human-machine interaction and fault tolerance. 